\title{
De la porosidad y otras expansiones de los cueppos*
}

\author{
Margarita María Zapata López \\ Universidad de Antioquia, Medellín, Colombia \\ margarita.zapatal@udea.edu.co \\ https://orcid.org/0000-0002-7108-0699 \\ Ediliberto Hernández González \\ Universidad San Buenaventura, Medellín, Colombia \\ edilberto.hernandez@usbmed.edu.co \\ https://orcid.org/0000-0002-6274-4078
}

\section{RESUMEN}

En este artículo nos adentramos en las tramas estéticas que dan soporte a la obra artístico-experimental Ascensos y pendulaciones, y las reflexiones que en torno de ellas hemos compuesto. Esta performance ha sido construida en correspondencia con la perspectiva de investigación denominada experimentación-creación con artes. En el contexto de un trabajo colaborativo entre la línea de investigación en Estudios Culturales y Lenguajes Contemporáneos, del Grupo de Investigación Esined de la Universidad de San Buenaventura, y el Grupo de Investigación Estudios en Educación Corporal de la Universidad de Antioquia. Entre los conceptos compuestos en el proceso, destacamos el encuentro sensible y creador donde el espacio ha sabido de nosotros y nosotros del espacio, hasta construir una atmósfera en la que nos hemos sabido juntos.

Palabras clave: experimentación-creación; cuerpos; cuerpos porosos; cuerpos expandidos; performance.

Cómo citar: Zapata López, M. y Hernández González, E. (2019). De la porosidad y otras expansiones de los cuerpos. Ciencias Sociales y Educación, 8(16), 43-56. DOI: https://doi.org/10.22395/csye.v8n16a3 Recibido: 10 de julio de 2019.

Aprobado: 15 de noviembre de 2019. 


\section{On Porosity and Other Expansions of the Bodies}

\section{ABSTRACT}

In this article, we deepen into the aesthetical threads supporting the artisticexperimental play Ascending and pendulations and the reflections composed by us around it. This performance has been built in correspondence with the Artistic Experimental-Creative research approach in the context of collaborative work within the Cultural Studies and Contemporary Languages line from the Esined research group from the Universidad
San Buenaventura and the Research Group on Body Education from Universidad de Antioquia. Between the concepts crafted in the process, we highlight the sensitive and creative encounter where space has known about us and we have known about it until we build an atmosphere in which we feel both known.

Keywords: experimental-creative; bodies; porous bodies; expanded bodies; performance.

\section{Da porosidade e outras expansões dos corpos}

\section{RESUMO}

Neste artigo, entramos em enredos estéticos que justificam a obra artístico-experimental Ascensos y pendulaciones, e as reflexões que surgiram sobre elas. Essa performance vem sendo construída em correspondência com a perspectiva de pesquisa denominada experimentação-criação com artes; no contexto de um trabalho colaborativo entre a linha de pesquisa em Estudos Culturais e Linguagens Contemporâneas, do grupo de pesquisa Esined da Universidad de
San Buenaventura, e o grupo de pesquisa Estudos em Educação Corporal da Universidad de Antioquia, ambas na Colômbia. Entre os conceitos compostos no processo, destacamos o encontro sensível e criador em que o espaço sabe de nós e nós do espaço, até construir uma atmosfera em que sabemos juntos.

Palavras-chave: experimentação-criação; corpos; corpos porosos; corpos expandidos; performance. 
Con tanto ser humano que se cuela por los poros de una piel como la nuestra, habrá formas menos enervantes de ser y estar en este mundo.

(Eugenia Prado Bassi, 2017, p. 9)

\section{Introducción. Hacia el abismo de un poro abierto}

La experiencia performática y la performance misma de la que nos ocupamos en este texto extienden sus raíces en las tramas de conocimiento fraguadas en torno a la investigación Cartografías de una educación (otras). De manera particular, el interés se dirige a los objetos, el cual fue desplegado en uno de los productos de esta investigación, un seminario con estudiantes de doctorado que tuvo por título los objetos, sus maneras de agenciar la vida y la investigación. En este seminario en el cual exploramos los vínculos del cuerpo con los objetos.

En el contexto de este seminario, nos detuvimos en la experimentacióncreación con objetos cotidianos, obras artísticas y creaciones escriturales, las cuales dieron paso a reflexiones colectivas y nuevas producciones que desembocaron finalmente en la configuración de la instalación Ascensos y pendulaciones exhibida en el Centro Cultural de la Universidad de Antioquia.

Entre los aspectos fortuitos que le dieron cuerpo a Ascensos y pendulaciones, tenemos un recorrido que hicimos con los funcionarios del Centro Cultural en el que consideramos los diversos espacios en los cuales podríamos realizar nuestro proyecto artístico. Nos hablaron de un espacio en desuso que nombran Sala Griega; este nombre y sus comentarios aumentaron nuestra curiosidad. Con estas sensaciones entramos al camerino de una sala de teatro ubicada al otro lado del corredor. Una vez atravesado el camerino, accedimos a un recinto ruinoso que, al percatarse de nuestra presencia, nos muestra toda su potencia.

Es un espacio angular de cubierta metálica y piso en concreto que hace las veces de techo para el auditorio ubicado en la planta inferior, el piso tiene un importante porcentaje de pendiente, lo que evoca los antiguos teatros griegos. Este lugar no nos deja indemnes. Una vez en él, nuestra percepción pierde sus referentes habituales y tenemos que lidiar con una superficie (piso) que se levanta y viene a nosotros potente y singular.

Durante unos instantes merodeamos la sala, ascendemos con dificultad, vamos de un lado a otro y, sin saberlo, lo irregular se va haciendo cuerpo en nosotros. Evocamos un pensamiento de Didi-Huberman (2015):

[...] en un intervalo minúsculo, ya se ha construido una complejidad. Lo que aparece, aparece de manera singular. Pero singularidad no quiere decir simplicidad, 
menos aún univocidad. Es singular lo que es irreductible y, por lo tanto, portador de extrañeza, noción también cargada de cierto desdoblamiento o duplicidad. (p. 85)

Entonces, la performance que componemos explora unos modos de experimentar, de jugar, de habitar en y con el espacio.

\section{Poros que suspiran: de la microscopía sensible a una percepción expandida}

Nuestro acercamiento al concepto de cuerpos porosos, inicia el camino en un punto microscópico necesario, preguntándonos qué son y qué hay en ellos. Podríamos hablar de lo poroso como una propiedad física de volumen que ocupa espacios vacíos y está próxima a la de densidad. Podríamos decir, por ejemplo, que es un conjunto de irregularidades entre partículas sólidas o el espacio volátil que fragiliza y hace poco consistentes a los cuerpos. En las redes porosas vemos aberturas, agujeros, ojos, perforaciones, cavidades, surcos, orificios, en fin, cualidades que no paran de aparecer. Pero los cuerpos porosos que nos interesan aquí no se agotan en sus formas, ya que vamos tras una porosidad que trastoca la característica para tornarla condición, estado, flujo, híbrido, posibilidad inagotable de lo corpóreo.

El cuerpo ha sido carne de estudio para muchos campos de conocimiento. En el transcurso de su historia, se le ha vigilado, corregido, disciplinado y castigado (Foucault, 2002); se le ha hecho dócil y maquinizado (Corbin, Courtine y Vigarello, 2006); pero también se le ha postulado como un asunto en constante reinvención (Butler, 2002).

Es de anotar que, igual que los campos de conocimiento, los contextos sociohistóricos también crean los cuerpos que necesitan para moldear y administrar la vida, adecuándola a cierta normalidad, prolongándola y compensando sus deficiencias. En 1979 lo expresó Foucault (2002) con la práctica del biopoder de los estados modernos y la "explosión de técnicas diversas y numerosas para obtener la sujeción de los cuerpos y el control de las poblaciones" (p. 169).

$\mathrm{El}$ éxito de estas prácticas ha hecho que permanezcan y se renueven en estrategias, como lo resume Castel (1984), con el propósito de "movilizar a los individuos expuestos a sus propias limitaciones, para que asuman la exigencia de administrarlas, convirtiéndolos en gestores de sí mismos" (p. 212). Como consecuencia, el biopoder resulta en la determinación de unos tipos de cuerpo y maneras de ser y aparecer en el mundo, maneras de subjetivación.

Ahora bien, estos postulados en los que históricamente se ha concebido al cuerpo en su dimensión más orgánica: cuerpo individual —anatómico, cuerpos de la población— biológico, se acompañan en la contemporaneidad de otras maneras de pensarlo e inventarlo. Hoy el cuerpo se nos presenta como inagotable 
y casi inasible, pues todo el tiempo se exige a sí mismo, otro. Lo que nos hace detener la mirada para recordar a Stelarc (1997):

\begin{abstract}
Llegó el momento de preguntarnos si un cuerpo bípedo, que respira, con visión binocular y un cerebro de $1.400 \mathrm{~cm}^{3}$ es una forma biológica adecuada. No puede con la cantidad, complejidad y calidad de las informaciones que acumuló: lo intimidan la precisión y la velocidad [...] El cuerpo no es una estructura ni muy eficiente, ni muy durable; con frecuencia funciona mal [...] Hay que reproyectar a los seres humanos, tomarlos más compatibles con sus máquinas. (p. 54)
\end{abstract}

La inquietud que plantea Stelarc, es una pregunta constante ante la cual nos tendremos que enfrentar a medida que se van transformando culturalmente las maneras, y se crean formas de existencia asociadas a los desarrollos tecnológicos. Este asunto lo ratifica Sibilia (2012) en su estudio de la tecnociencia, al exponer que los cuerpos contemporáneos "en su condición biológica, se estarían volviendo obsoletos [...] en tanto se presentan como sistemas de procesamiento de datos, códigos, perfiles cifrados, bancos de información" (p. 13).

Pero no son solo los cuerpos biológicos los que están siendo cuestionados, también las conceptualizaciones socio-antropológicas clásicas en torno a los cuerpos entraron en crisis, ya que la mirada contemporánea del cuerpo convoca necesariamente una perspectiva transdisciplinar, dada la condición inasible (porosa) del cuerpo como construcción epistemológica. Pensarlo como un lugar definido y quieto ya no es insuficiente. Es por eso que una comprensión del cuerpo convoca justamente la exploración de otros saberes del cuerpo y desde el cuerpo mismo, que, si bien están cercanos a su propia organicidad —a las redes, cavidades y universos que contiene y lo que circula por ellos-, cruzan la frontera para aparecer siempre otros.

Este abordaje es en cierta medida contrahegemónico, porque irrumpe en lo establecido (que creemos también inestable) y viene a producir unos cuerpos que no logran contener su aparente solidez, sino que se derraman por sus agujeros, se desplazan en el espacio, pero el espacio mismo los penetra. Lo que aparece aquí es un cuerpo viscoso que se adhiere a todo lo que toca. No es un cuerpo orgánico, pero tampoco exclusivamente un cuerpo virtual o el cuerpo sin órganos que trajeron Deleuze y Guattari de Artaud: no es un cuerpo dios. Sin embargo, es "divino el carácter de su energía de disyunción" (Deleuze, 1974, p. 21).

$\mathrm{El}$ cuerpo que nos interesa o mejor, los cuerpos que interesan a nuestra performance son cuerpos plurales, inscritos en disyuntivas, intersticiales, irregulares, diversos y múltiples, profundamente porosos para hacer espacio al vacío, a otros cuerpos y a sí mismos, poniendo a prueba otras maneras de aparecer. Son cuerpos-vacío: "Incluso el vacío es una especie muy sutil de cuerpo" (Nancy, 2016, p. 105) y cuerpos-pensamiento: "El yo pensante se estremece a lo largo de la espina dorsal: yo pienso por todas partes" (Serres, 2003, p. 96). 
Y esos cuerpos son los que nos han hablado, los que se nos han presentado en nuestro proceso de experimentación-creación. Pero tanto para saberlos, como para dar cuenta de ellos, requerimos de una cierta sensibilidad que nos colocará en donde propone Suely Rolnik (1987) —refiriéndose a las cartografías-, como una "adyacencia de las mutaciones" que se vale de un "compuesto híbrido" (p. 2) hecho por nuestros ojos, y nuestros cuerpos vibrátiles, experimentando la tensión fecunda entre flujo y representación, para canalizar las intensidades y darles sentido.

Mirar, tocar, ser, escribir los cuerpos porosos desde la experimentacióncreación, amerita ponerse in situ; dejarse atravesar, expandirse, inhalar y exhalar otros cuerpos, desde una disposición sensible y atreverse incluso a desaparecer de sí mismo para devenir otra cosa, otro cuerpo. Al respecto, Castro (2016) refiere el cuerpo poroso como:

[...] un estado corpóreo-subjetivo de permeabilidad, condición para desfigurar la experiencia de sí y hábitos pre-establecidos. [...] no es una permeabilidad lisa, sino una disposición tensada por el riesgo de ser alterada o desplazada por las fuerzas del mundo, del otro y que no deja indemne el modelo de la acción y de la percepción de uno mismo. (p. 276)

Julia Castro centra su atención en lo poroso, en tanto condición de permeabilidad corporal, lo que nos alienta a pensar en un cuerpo que está al margen de toda posibilidad de identidad ontológica. En este sentido, nuestra performance necesitó de un cuerpo que se vio forzado a detener el desplazamiento, de manera que al participar de esa aparente condición de no ir para ninguna parte, se activaran otras intensidades, unas que ya no se componen de desplazamientos sino de vibraciones.

Para esta performance nos interesaban los cuerpos que ocupan el espacio, y que justamente lo performan, a fin de producir cuerpos donde lo volátil ocupe mayor espacio. En esta experiencia performática inauguramos cuerpos ramas, cuerpos enraizados, de movimientos diminutos, casi imperceptibles, y que en su forma de acción se apuntalaran al espacio, de manera que en sus desplazamientos arrastraran consigo al espacio que los contenía.

Los cuerpos porosos que ponemos aquí, son nacidos de la experimentación con la performance. Al respecto, nos inspiran las reflexiones históricoconceptuales de Taylor y Fuentes (2011), en el sentido que una performance propone diversidad de ensambles y composiciones de la "re-presentación, con la transmisión del conocimiento a través de gestos corporales, con la mirada del espectador" (p. 12), y un singular encuentro con y en el espacio, es decir, el espacio en la performance deviene también poroso.

En esta misma línea María José Arjona, citada por Sánchez (2018), se pregunta: "¿qué le ocurre al cuerpo en la duración?", y plantea además que encuentra en 
la performance "un territorio en que podía seguir haciendo, haciendo, haciendo para ver qué otras fuerzas, qué intensidades, qué estados físicos podían salir de allí" (Sánchez, 2018).

Ahora bien, una experiencia artística que necesita y construye cuerpos porosos (poríferos), permeables (espongiarios), expandidos, hidratados; cuerpos que se contraen, que dejan entrar y salir, captan, construyen; cuerpos como tejido, espuma, red, nos mueven e invitan a pensarnos por fuera de las dicotomías occidentales que han fundamentado la percepción de nosotros mismos. En esta tarea — valga decir — no solo las artes nos venimos ocupando, sino también en el campo de la antropología Roy Wagner (1991) propuso el concepto de "personalidad fractal" para pensarse unos modos de presencia más integrativos (p. 163).

Deleuze, por su parte, plantea que la univocidad del ser no quiere decir que haya un solo y mismo ser. Al contrario, los entes son múltiples y diferentes, producidos siempre por una síntesis disyuntiva, disjuntos y divergentes ellos mismos, membrana disjoncte. La univocidad del ser significa que el ser es voz, que se dice, y se dice en un solo y mismo "sentido" de todo aquello de lo que se dice (Deleuze, 1974).

Las prácticas y elaboraciones teóricas de las artes, la antropología y la filosofía, nos van dando pistas para los cuerpos que vamos componiendo en cada experiencia de experimentación-creación, que nos remiten a pensar si continuamos siendo sujetos o tenemos subjetividad. A esta pregunta podría respondernos Silvia Citro, en su conferencia taller cuando afirma: "somos el efecto acumulado de sucesivas prácticas de subjetivación" (comunicación personal, mayo de 2019). Tal vez en ese mismo existir cor-póreo (cuerpo-poro) puesto ahí de manera performativa y performática, lo que hemos tenido por subjetivación desaparece, se deshace y rehace siempre en relación, es flujo y pálpito.

Nos encontramos frente a una realidad movediza pero no frágil, de unas subjetividades flexibles ${ }^{1}$, referidas por Rolnik (2009), en las que la propia existencia se da por la existencia del otro, de lo otro. En tanto se experimentan modos de existencia, se reinventan relaciones y se incorporan "fuerzas que agitan [el propio] entorno". En este sentido, el cuerpo es un cuerpo que "se afecta por la alteridad del mundo" y deviene otro de sí mismo permanentemente.

1 La noción de "subjetividad flexible" está desarrollada en varios ensayos, entre los que se encuentra "Politics of Flexible Subjectivity. The Event-Work of Lygia Clark", en Terry Smith, Nancy Condee \& Okwui Enwezor (eds.), Antinomies of Art and Culture: Modernity, Postmodernity and Contemporaneity, Duke University Press, Durham, 2006; "Life for Sale", en Adriano Pedrosa (coord.), Farsites: urban crisis and domestic symptoms. InSite, San Diego y Tijuana, 2005. Véase Brian Holmes, "The Flexible Personality", en Hieroglyphs of the Future, WHW y Arkzin, Zagreb, 2002), accesible online en <http://www.u-tangente.org/> [versión castellana: "La personalidad flexible. Por una nueva crítica cultural”, en, Brumaria, n. ${ }^{\circ}$ 7, Arte, máquinas, trabajo inmaterial, 2006 (http:///www.brumaria.net) y publicación multilingüe en este monográfico, transversal: máquinas y subjetivación. <http://transform.eipcp.net/transversal/1106/holmes/es>]. 
A propósito de la performance que nos provocó los poros de este texto, el acercamiento a las subjetividades flexibles de Rolnik (2009) nos permitió nuevas miradas en relación con el arte contemporáneo, en las que cambia:

[...] El régimen de la creación artística que ya no es de cierre, de protegerse a sí mismo, sino que son prácticas que envuelven al otro y donde la obra que se hace entra en esa relación y ya no solo fetichizada en el objeto de arte. (Colectivo Situaciones, 2006)

Tanto la performance como los cuerpos que la hacen posible, constituyen superficies porosas y a través de sus orificios es posible dirigirnos al reverso de las cosas, espacio que nos devuelve un gesto dactilar creado en el roce con esas mismas superficies. Es entonces cuando queremos tentar, acariciar con todo el cuerpo, pensar con todos los poros. Tocar por primera vez.

\section{De la experimentación-creación a un devenir cor-póreo}

Nuestros procesos de experimentación-creación, parten de un esfuerzo por detener ciertos organizadores de la realidad y formas de comprensión del cuerpo, para que el universo de lo discontinuo tenga lugar. Este movimiento de lo corpóreo es paralelo a la pérdida de la potencia de la representación, experimentada en las artes contemporáneas en el sentido de que los cuerpos y los objetos en acción artística no están allí para indicar otra cosa. Los objetos, palabras, acciones, sensaciones devienen en el espacio - público o de exhibición-justamente para ser aquello que dicen ser. No queremos negar con ello la polifonía propia de las cosas, pero su pluralidad de sentidos no se articula mediante una lógica discursiva que acentúe la coherencia que las definen. En la experimentación los sentidos se superponen, a veces se amarran y otras veces permanecen sueltos o levemente atados; es importante que los sentidos se presenten apenas zurcidos.

Hemos creado unas condiciones para la performance que nos exigió explorar los límites mismos del cuerpo, obstruir unos conductos y las potencias que circulaban por ellos, para que el cuerpo experimentara nuevas expansiones. La performance Ascensos y pendulaciones constituyó un gesto estético creado en conversación con un espacio que reclamaba un cuerpo que reptara por él (figura 1), y el cuerpo se descubrió poroso para corresponder a la inclinación de ese espacio. 


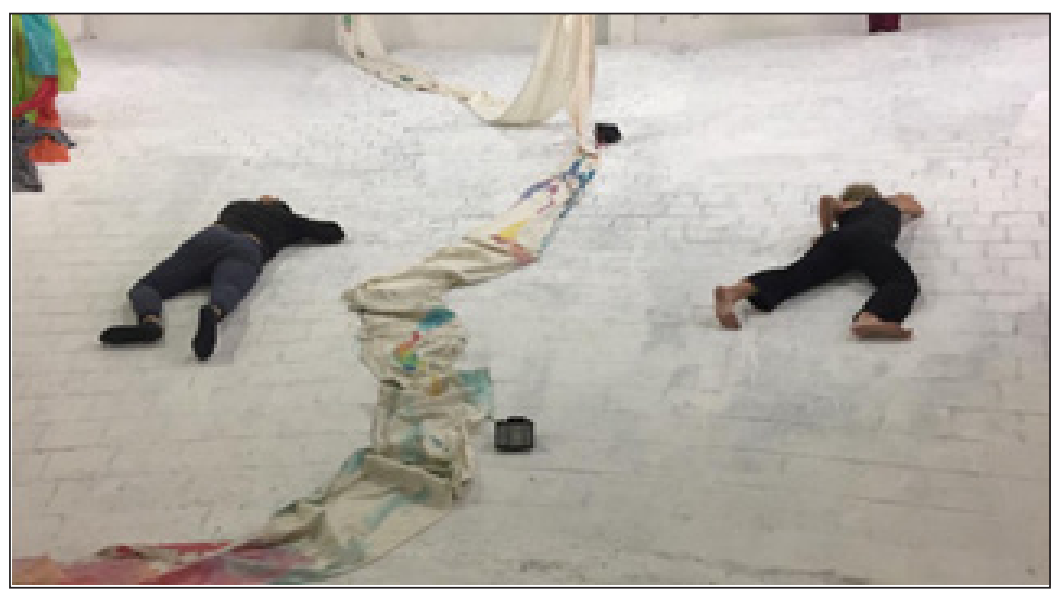

Figura 1. Ascensos y Pendulaciones

Fuente: archivo personal, Juan Fernando Castaño, Medellín, 2018.

La performance Ascensos y pendulaciones propuso un movimiento vibrante que asciende en su propia inmovilidad. En ella pusimos en acción una secuencia de movimientos pesados, cuyas fuerzas principales conducían a habitar el movimiento mismo. La afirmación de André Lepecki (2011) que dice que "en el punto inmóvil que no es fijeza ni movimiento yace la inmovilidad" (p. 527) abre un interesante camino a través del cual nos preguntamos: ¿Cuántas capas de espacio y de tiempo es capaz de ocupar un cuerpo? De alguna manera, la respuesta tuvo que ver con los límites que dibujó la propia experimentación.

Por ello, buscamos forzar nuestros cuerpos a reducir al mínimo sus desplazamientos para que estos se hicieran espacio, para que se hicieran tiempo. Esta performance accionó un cuerpo inflamado, capaz de experimentar otras intensidades del movimiento en que el espacio fuera agujereado de manera que el cuerpo pudiera entrar y salir por esos agujeros. Una vez iniciada la performance, las nociones del transcurrir cronológico (una hora y veinticinco minutos) desaparecieron, solo los espectadores sabían de este paso del tiempo.

Así, nuestra performance, en tanto acción en el presente, terminó por agujerear el tiempo. José Luis Pardo (1991), en su abordaje del trabajo de Cézanne, reitera que lo que hace el artista es "pintar el tiempo, pero no el tiempo como sucesión de in-stantes, sino ese otro tiempo que no sucede, que no se localiza en la serie de los momentos, la forma de lo que pasa, que es lo que no pasa" (p. 78).

Y quizás sea esto lo que buscamos siempre: un agujero por el cual bucear para sentir que estamos vivos y escapar por momentos a la dictadura de un tiempo que pasa sobre nosotros rasgando la piel. Pero también cabe decir que los agujeros conducen a otros espacios o tiempos de "felicidad" aparentemente 
sin orificios; a un cuerpo-sensación, todo expansión, todo humedad, vapor que recorre cavidades y no retorna, un cuerpo-vacío, uno que ya no es cavidad, sino ausencia de superficies contenedoras. ¿Qué puede contener un espacio que se ha perdido? ¿En qué contener esas laderas que se vienen abajo y la corriente se lleva?

Esta condición de fluidez se torna vital para la performance, la nuestra, en tanto hizo una renuncia premeditadamente a la postura erecta, tan valorada en los discursos evolucionistas occidentales, para hacer presente un movimiento ondulatorio compuesto de contracciones y extensiones que le permitieran a los cuerpos desplazarse sobre una superficie con un veinte por ciento de pendiente. Este modo de ascenso exploró una estética-reptil (figura 2), que nos puso en contacto con la intimidad de las superficies. Reptamos para hacernos espacio con el espacio, acortamos toda distancia, estuvimos cuerpo a cuerpo con el espacio, tirados y abandonados en el espacio, mientras de forma intermitente se escuchaba la voz de la soprano cantando: "deja que llore mi cruel suerte y que suspire la libertad", aria del segundo acto de la ópera Rinaldo de Georg Friedrich Händel ${ }^{2}$.

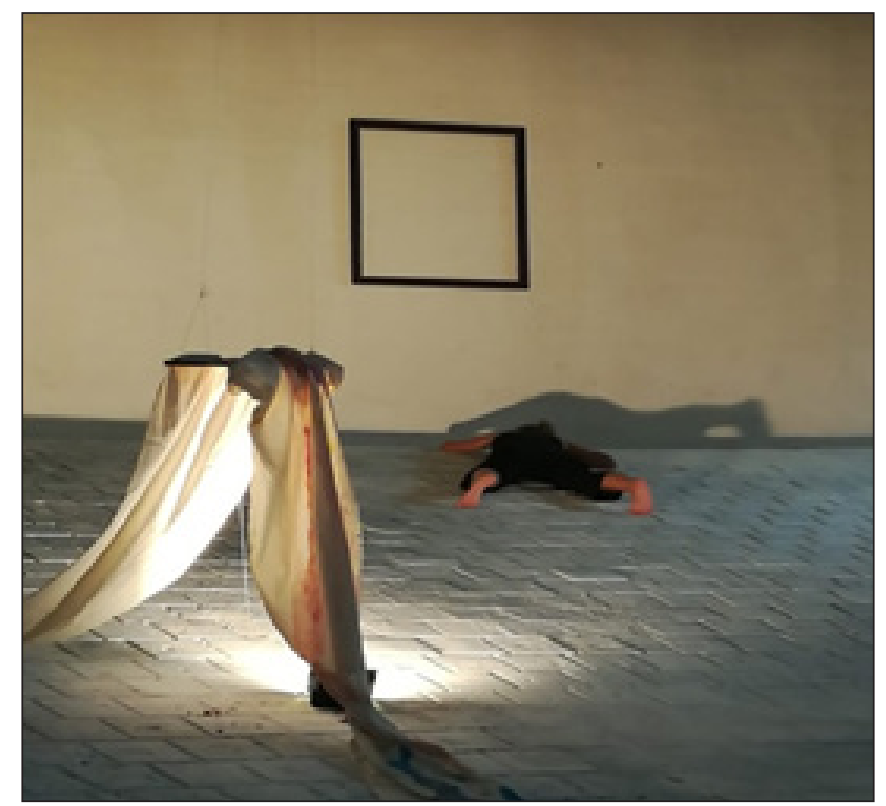

Figura 2. Ascensos y pendulaciones

Fuente: archivo personal, Juan Fernando Castaño, Medellín, 2018.

2 En la performance el aria fue interpretada por la soprano brasileña Fernanda Keiko Miki da Costa. Possui graduação em Música - Canto pela Universidade Federal de Pelotas (2018). 
En esta performance, el cuerpo se olvidó incluso de sí mismo. Las potencias corporales cesaron para que el peso - del cuerpo mismo y del mundo- ocupara todos los poros; cada vez estábamos más adheridos al piso y a los muros. Una lluvia huracanada repicaba sobre las placas metálicas de la cubierta. Aquellas presencias eran también gotas de cuerpo que reclamaban su propio espacio, unas veces tibias y gratas, otras ardientes y penetrantes; luego supimos que esta lluvia corporalmente experimentada y amplificada solo se fue una leve llovizna.

Es importante anotar que si bien, nuestra creación performática no tuvo un carácter explícitamente político ni reivindicatorio, tampoco fue ajena ni desconoció la realidad de miles de personas que permanecen día y noche olvidadas en las calles y andenes de nuestras ciudades. En el caso de Medellín, el documento Política pública social para el habitante de calle, el Ministerio de Salud registra entre 2009 y 2016 un total de 2.721 habitantes de calle $(2018$, p. 35). Estas cifras contrastan con el informe del Centro de Estudios de Opinión, de la Universidad de Antioquia, realizado para la Secretaria de Bienestar Social de la Alcaldía de Medellín, en el cual se concluye que en la cabecera municipal y sus corregimientos: "En total se censaron 24.352 habitantes en situación de calle distribuidos así: el $13,88 \%$ son habitantes de calle y $86,12 \%$ son habitantes en calle" (2009, p. 46). Estos datos no solo difieren notablemente de un informe al otro, sino que, resultan alarmantes en cualquiera de las dos circunstancias.

\section{De los cuerpos que reptan ${ }^{3}$}

Entregarse al piso, donar el peso al piso, ser el piso. El piso me abraza, quiere todo de mí, me posee y ejerce celosamente su fuerza para que yo me pese. Entro por los canales de las pizarras, el piso se hace grieta y me traga. Ahora me torno grieta. Somos un solo laberinto de grietas y orificios, vacío y bordes. El cuerpo pesa, casi inmóvil, es un flujo sólido, intenta descorrerse en desafío. Devengo concreto gastado, un cuerpo duro, frío, húmedo y viejo. Somos un piso que se desvanece a un mismo ritmo de respiración, hablamos a una sola voz.

Escucho los latidos, mi cuerpo salta, pero es el piso quien ha empezado a latir. Soy toda expansión. No sé qué parte del piso soy o si el piso en su completud soy yo. No sé si el piso es yo, o él es una parte mía. Angustia, identidad de partículas no densas. ¿Qué acontece en este intermezzo entre el piso y yo? Somos una sola cosa, multipolar tal vez. ¿Hay un devenir que escapa aún de mi comprensión? "Mi Dios, mi Dios, ¿a quién asisto? ¿Cuántos soy? ¿Quién es yo? ¿Qué es este intervalo que hay entre mi persona y yo?" (Pessoa, 2007, p. 219).

En esta sección invitamos al lector a participar de la experiencia íntima que se produjo en la performance. El uso de la primera persona quiere contribuir a tal intimidad. 
Los objetos y el espacio entraron por los poros del cuerpo (figura 3). Todo el cuerpo fue un poro sin paredes que le obstruyeran la salida. En estas expansiones y contracciones en las que el cuerpo se debate, esos objetos, ese espacio, se hizo otros cuerpos ¿Heterocorporalidades? iTal vez! El piso-muro no paró de recorrerme, fui piso, fui muro y no sé si ese cuerpo gélido fue suyo o mío. Salir del piso no fue dejar de aparecer piso, el piso se fue conmigo. Soy ahora un piso que camina. Un recorrido asfáltico que se niega terminar.

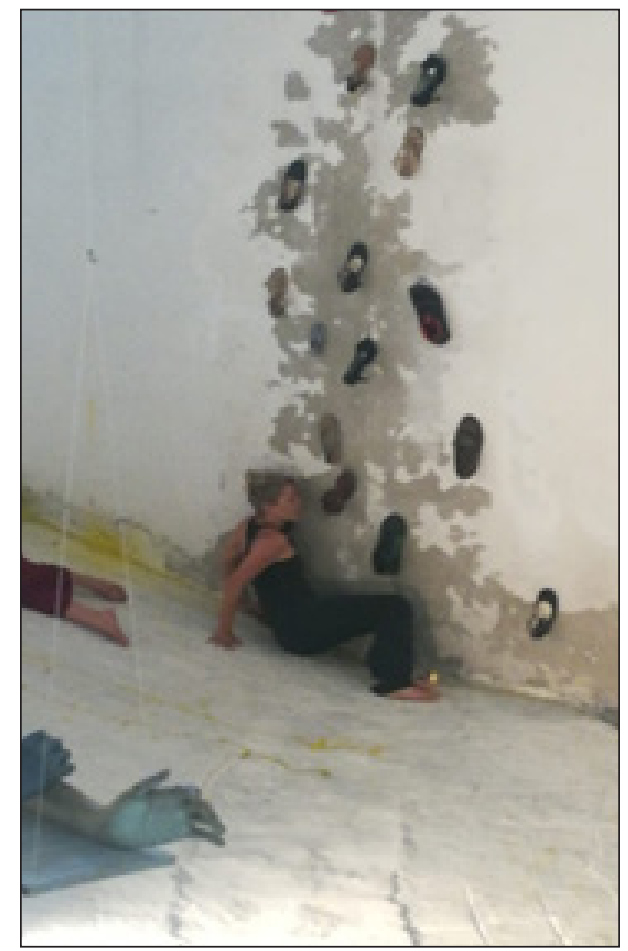

Figura 3. Ascensos y pendulaciones

Fuente: archivo personal, Juan Fernando Castaño, Medellín, 2018.

\section{De lo inconcluso}

La performance crea condiciones corporales capaces de producir una experiencia estética, a la vez que busca subvertirla. Nuestra performance surge en medio de los interrogantes por los objetos, por sus formas de aparecer, por sus lenguajes y por lo que ellos tienen por decir a nuestros modos de subjetivación contemporáneos. En este sentido, nos preguntamos por algunas condiciones vinculares con los objetos y con el espacio: ¿Qué le dice un cuerpo que repta en la intimidad con el suelo a ese sentido hegemónico de posesión y a la industria que lo sustenta a base de titulaciones y seguros de propiedad? Si nos hemos 
hecho seres de lenguaje, ¿cuáles son esos matices que hablan en un encuentro donde pareciera que el cuerpo y el espacio se penetran a sí mismos y se pierden el uno en el otro para devenir un-otro?

El cuerpo o los cuerpos expuestos son espacio performante y performador, dislocan, crean y mutan; siempre están yendo a otros lugares, puesto que son y pueden ser otra cosa. Es por ello que Ascensos y pendulaciones nos remitió a la pregunta por esas subjetividades encarnadas que hemos llegado a comprender desde distintas formas de aparecer en el mundo. No afanaremos respuesta, porque ya en sentido cor-póreo supimos que también aparece como múltiple y porosa.

Disponerse para que algo pase, abandonarse cuerpo-concepto, partir sin saber a dónde se llega o si se llega, desmontar las narrativas que han intentado definir lo que puede un cuerpo, haciéndose cuerpo poroso, ratifica la multiplicidad y la infinitud de los cuerpos como campo de experimentación y creación. Y esta cercanía e intereses comunes en torno a la configuración de materializaciones a partir de las experiencias de investigación en las que el cuerpo y los objetos constituyen una presencia potente, nos han posibilitado aproximarnos y poner en circulación esos saberes producidos en el proceso. El espacio y los objetos han sabido de nosotros y nosotros del espacio y los objetos. Nos hemos sabido juntos.

\section{Agradecimientos}

Este artículo es derivado de la investigación Cartografías de una educación (otras), la cual hizo parte de los proyectos seleccionados y financiados en la convocatoria de la Dirección de Investigaciones de la Universidad de San Buenaventura, Medellín, 2017-2018.

\section{Referencias}

Butler, J. (2002). Cuerpos que importan: sobre los límites materiales y discursivos del "sexo". Buenos Aires: Paidós.

Castel, R. (1984). La gestión de los riesgos: de la anti-psiquiatría al post-análisis. Barcelona: Anagrama.

Castro, J. A. (2016). Corporalidades sensibles y subjetividades corporizadas en Medellín, 1980-2012 (tesis doctoral en Ciencias Sociales). Universidad de Antioquia, Medellín, Colombia. Recuperado de: https://bit.ly/39bwvns

Citro, S. (junio de 2015). Provocaciones interculturales sobre cuerpo, subjetividad y saber. Encuentro Internacional Diálogos Interculturales de la danza de y desde los cuerpos. Conferencia y Taller de Experiencia Práctica llevado a cabo en el encuentro de Flacso, Ministerio de Cultura y Patrimonio, Campaña Nacional de Danza del Ecuador, Quito, Ecuador.

Colectivo Situaciones. (2006). Entrevista a Suely Rolnik. Revista Mu Lavaca, 4. Recuperado de https:// bit.ly/2I4GO0y 
Corbin A., Courtine J. J. y Vigarello, G. (2006). Historia del cuerpo. Volúmenes I, II y III. Las mutaciones de la mirada. El siglo XX. Madrid: Taurus.

Deleuze, G., y Guattari, F. (1974). El antiedipo. Barcelona: Barral.

Deleuze, G. (1994). Lógica del sentido. Barcelona: Paidós.

Didi-Huberman, G. (2015). Falenas. Ensayos sobre la aparición. Santander: Asociación Shangrila Textos Aparte.

Foucault, M. (1977). Historia de la sexualidad. Vol. 1. La voluntad de saber. Madrid: Editorial Siglo XXI.

Foucault, M. (2002). Vigilar y castigar. Nacimiento de la prisión. México: Editorial Siglo XXI.

Lepecki, A. (2011). Inmóvil: sobre la vibrante microscopía de la danza. En: Taylor, D, y Fuentes, M. Estudios avanzados de performance (pp. 521-548). México D. F: Fondo de Cultura Económica.

Ministerio de Salud (2018). Política pública social para el habitante de calle. Recuperado de https:// bit.ly/32xVq1W

Nancy, J. (2016). 58 indicios sobre el cuerpo. En Corpus. Madrid: Arena Libros.

Pardo, J. (1991). Sobre los espacios, pintar; escribir; pensar. Barcelona: Ediciones del Serbal.

Pessoa, F. (2007). Libro del desasosiego. Traducción de Santiago Kovadloff. Buenos Aires: Emecé Editores.

Prado, E. (2017). Advertencias de uso para una máquina de coser. Santiago de Chile: Editorial Carnicera.

Rolnik, S. (1987). Cartografia ou de como pensar com o corpo vibrátil. São Paulo: Núcleo de Estudos de Subjetividade da PUC.

Rolnik, S. (2009). Políticas del fluido híbrido y flexible. Para evitar falsos problemas. Nómadas, (31), 157-164.

Sánchez, F. (2018). El cuerpo desfigurado. Revista Arcadia, (158). Recuperado de https://bit.ly/3ch3jgF

Serres, M. (2003). Los cinco sentidos: ciencia, poesía y filosofía del cuerpo. México: Editorial Taurus.

Sibilia, P. (2012). El hombre postorgánico: cuerpo, subjetividad y tecnologías digitales. México: Fondo de cultura económica.

Stelarc. (1997). Das estratégias psicológicas às ciberestratégias: a protética, a robótica e a existência remota. A arte no século XXI, a humanização das tecnologias, org. En: D. Domingues, São Paulo, Ed. Unesp.

Taylor, D., y Fuentes, M. (2011). Estudios avanzados de performance. México: Fondo de Cultura Económica.

Universidad de Antioquia, Centro de Estudios de Opinión. (2009). Censo de habitantes de calle y en calle de la ciudad de Medellín y sus corregimientos. Revista electrónica, La Sociología en sus escenarios, 21 (13). Recuperado de https://bit.ly/2VuTLsr

Universidad de Antioquia, Centro de Estudios de Opinión. (2009). Revista electrónica, La Sociología en sus escenarios. 21 (13). Recuperado de https://bit.ly/32BGUGq

Wagner, R. (1991). The fractal person. En: M. Strathern y M. Godelier (eds.), Big men and great men: personifications of power in Melanesia. Cambridge: Cambridge University Press, New York. 